\title{
Novos meios e novos métodos de pesquisa: desafios, soluções e avanços
}

\author{
New means and new methods of research: \\ challenges, solutions and advances
}

Nuevos medios y nuevos métodos de investigación: desafíos, soluciones y avances

\section{Leandro Leonardo Batista}

- Doutor em Comunicação Social e mestre em Propaganda pela University of North Carolina

- Professor da Escola de Comunicações e Artes da Universidade de São Paulo (ECA USP)

- $\quad$ na graduação em Publicidade e na Pós-Graduação em Ciências da Comunicação

- Tem experiência acadêmica e profissional na área de Comunicação, com ênfase em Relações Públicas e Propaganda

- Líder do 4C - Centro de Comunicação e Ciências Cognitivas, da ECA-USP

- E-mail: leleba@usp.br

\section{Clotilde Perez}

- Pós-doutora em Comunicação pela Universidad de Murcia, Espanha

- Livre-docente em Ciências da Comunicação pela Escola de Comunicações e Artes da Universidade de São Paulo (ECA-USP)

- Doutora em Semiótica pela Pontifícia Universidade Católica de São Paulo (PUC-SP)

- Mestre em Administração de Marketing pela PUC-SP

- Professora da ECA-USP, na graduação em Publicidade e na Pós-Graduação em Ciências da Comunicação

- $\quad$ Professora da PUC-SP

- Presidente da ABP2 - Associação Brasileira dos Pesquisadores em Publicidade

- Fundadora da Casa Semio

- E-mail: cloperez@terra.com.br 


\section{Resumo}

0 presente artigo oferece reflexão sobre a evolução dos novos meios de comunicação e suas linguagens, prioritariamente na esfera digital, e seus impactos em métodos e técnicas de pesquisa de mercado. Problematiza a questão a partir de dois segmentos fundamentais à investigação: cobertura e respostas, considerando os novos desafios que a vida tecnologizada pelo digital constrói, bem como os avanços e as melhorias no planejamento, na execução e na análise das pesquisas. Ao final, discorre acerca das questões éticas concernentes ao tema e das limitações inerentes à reflexão apresentada.

\section{PALAVRAS-CHAVE: MÉTODOS •PESQUISA •PESQUISA DE MERCADO・DIGITAL・LINGUAGEM.}

\section{Abstract}

The present article debates the evolution of the new media and their languages, mainly in the digital sphere, and their impact on market research methods and techniques. The question is discussed from two fundamental research segments: coverage and responses, considering the new challenges brought by digitalised life, as well as the advances and improvements in the planning, execution and analysis of the researches. In the end, it discusses the ethical issues concerning the theme and the limitations inherent to the reflection presented.

\section{KEYWORDS: METHODS •RESEARCH・MARKET RESEARCH・DIGITAL・LANGUAGE.}

\section{Resumen}

En este artículo se ofrecen reflexiones sobre la evolución de los nuevos medios de comunicación y sus lenguajes, principalmente en elámbito digital, y sus impactos sobre los métodos y técnicas de investigación de mercado. Se problematiza el tema a partir de dos segmentos principales de investigación: la cobertura y respuestas, teniendo en cuenta los nuevos desafíos que la vida tecnologizada por lo digital viene construyendo, así como los avances y las mejoras en la planificación, en la ejecución y en el análisis de las investigaciones. Por último, se habla de las cuestiones éticas relacionadas con el tema y las limitaciones inherentes a la reflexión presentada. 


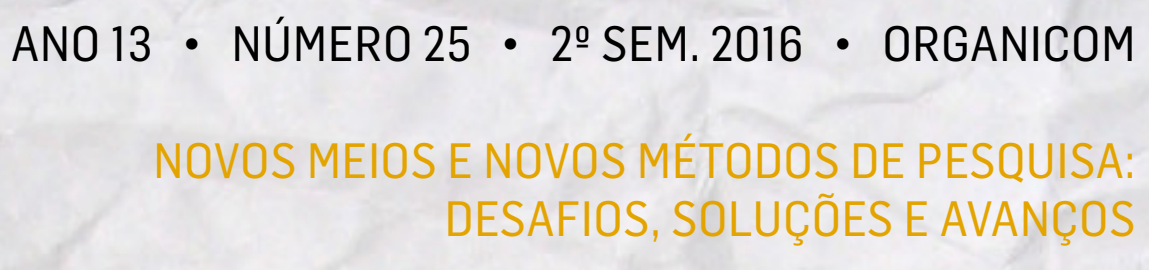

m um mundo que aglutina a rapidez, a profundidade e a transversalidade das mudanças, fica praticamente impossível entender o presente a partir do passado conhecido e ainda mais difícil projetar o futuro, dadas tantas incertezas. Esse contexto de imensa volatilidade e insegurança trouxe consequências profundas para o entendimento das pessoas, de seus comportamentos, suas atitudes e seus valores sociais. Tudo pode ser efêmero e estar em trânsito (LIPOVETSKY, 2008), o que imprime novas formas para buscar o entendimento das dinâmicas sociais e seus impactos cotidianos.

A tradição desta tentativa de compreensão, quando a ênfase está nas relações comerciais e de consumo, é da pesquisa de mercado. A pesquisa de mercado, também chamada de pesquisa aplicada, cresceu à medida que a competição entre empresas, marcas e produtos também aumentou. Podemos localizar este movimento mais intenso a partir da $2^{2}$ Guerra Mundial e o crescimento dos mercados e meios diversificados de comercialização. É evidente que a busca pelo conhecimento é inerente ao humano, o que implica que desde sempre esteve presente na espécie. No entanto, o foco nos processos de interação comercial realmente cresceu mais recentemente.

Outro fator que impulsionou o crescimento da pesquisa de mercado tem relação direta com a complexidade das identidades contemporâneas (LIPOVETSKY, 2008; CANEVACCI, 2008, HALL, 2004) e a transversalidade da tecnologia digital na vida cotidiana (JENKINS, 2009; JENKINS, FORD e GREEN, 2015).

Causa e consequência desse crescimento é o movimento de expansão da individualidade. Cada indivíduo é um e, portanto, precisa e quer protagonizar sua vida, com imensos reflexos em todas as áreas e segmentos, quiçá ainda mais nas ações de consumo, antes totalmente circunscritas às ofertas que se apresentavam. Customizar, personalizar são alguns dos caminhos que já evidenciavam o desejo genuíno de interferir nos processos até então passivos. Mais recentemente, consumo autoral, consumo performático, prosumidor, consumidor autor, fã etc. (PEREZ, 2004, 2015), são alguns dos conceitos que buscam apreender essas características performáticas e identitárias dos consumidores e cidadãos contemporâneos.

A pesquisademercadoéumconjunto demétodose técnicascientíficas (diferentedeumamontoadodeimprovisações, suposições e conhecimentos genéricos), que permite coletar, conhecer, experimentar e avaliar aspectos relacionados aos produtos, às empresas, aos serviços e aos indivíduos considerando suas formas, conteúdos, intenções, aspirações e comportamentos. Dentro dessa perspectiva estuda, analisa, avalia desde indivíduos em grupo (tais como famílias, lares, associações, trabalho) ou isoladamente, até outras unidades como empresas e entidades de uma forma geral ou mesmo produtos gerados por estes segmentos, tais como a comunicação organizacional, o design de produtos e serviços e a comunicação publicitária.

Dentro desse esforço, a pesquisa de mercado busca determinar características individuais, sociais e/ou culturais e fornecer orientação e caminhos alternativos para as tomadas de decisão em negócios e políticas de uma forma geral. Utiliza para isso questionários estruturados, conversas informais, reuniões individuais e em grupos, observação metódica (ou não), observação participante, método semiótico e avaliação de efeitos partindo de uma base sólida de métodos desenvolvidos desde pelo menos a metade do século passado. A aplicação desses métodos vem sendo adaptada, desde então, considerando o desenvolvimento dos meios pelos quais eles se corporificam e podem ser aplicados.

Os métodos, basicamente, se dividem entre qualitativos e quantitativos, sendo que ambos sofreram nas últimas décadas um ataque forte em suas bases metodológicas devido aos desenvolvimentos tecnológicos. Inicialmente a área quantitativa teve um maior número de aplicações das novas tecnologias, por exemplo, combinando computador e telefone, sendo que mais recentemente a pesquisa qualitativa também incorporou novidades tecnológicas que alteraram profundamente as opções para este tipo de pesquisa como, por exemplo, a discussão em grupo virtual e on-line. 
Assim, partiu-se de métodos bastante limitados e custosos, como, por exemplo, ir de casa em casa para as pesquisas quantitativas ou a obrigatoriedade de participantes de grupos de discussão estarem no mesmo local no mesmo momento, para uma nova situação onde dados mais rápidos, mais baratos, mais frequentemente coletados e de formas diversas causaram e ainda causam uma revolução nos métodos de pesquisa.

A partir dessas reflexões que envolvem os contextos de produção e interação, o presente texto visa apresentar discussão centrada na interação de métodos tradicionais de pesquisa de mercado com possibilidades, benefícios e deficiências oferecidas pelos meios recentemente introduzidos no mundo da pesquisa de mercado. Adicionalmente, busca apontar caminhos inovadores de métodos e técnicas que estão em construção e uso mais recentemente e que são rentáveis à investigação das relações e interações de consumo.

\section{ASPECTOS ASSOCIADOS AO MEIO}

\section{Efeitos na cobertura}

Os erros de cobertura, embora sejam mais importantes em pesquisas quantitativas, atingem toda a área que lida com a obtenção de informação, pois eles focam na porcentagem da população que pode ou não ser atingida devido ao modo escolhido para encontrá-la. Por exemplo, uma pesquisa por telefone tem um limite óbvio associado ao meio de coleta de dados que não se restringe apenas aos que não têm acesso ao meio, mas também à limitação de interação entre entrevistador e entrevistado. Mas só o primeiro é considerado erro de cobertura. Também na pesquisa qualitativa temos este erro de cobertura, mas os novos meios, nesse caso, ao invés de limitarem, podem expandir a abrangência, reduzindo o erro. Por exemplo, a participação em discussões em grupo pode ser ampliada pelo uso da internet (por exemplo, utilizando o Skype ou o Facetime). Assim, as pessoas que ou não tinham tempo ou facilidade de deslocamento para os pontos onde normalmente essas pesquisas são realizadas, agora podem participar a distância.

Como vimos, podemos considerar que os erros de cobertura, que sempre existiram, tomam hoje outra dimensão e devem ser considerados na definição de um meio para coleta de dados. Por exemplo, pessoas sem telefone, endereços que não recebem cartas, pessoas que só andam de carro, pessoas sem acesso à web, moradores de prédios e/ou condomínios etc. têm sua participação reduzida em pesquisas de mercado, tanto qualitativas quanto quantitativas, embora em menor escala nestas últimas, gerando vieses na determinação da população sendo observada, de onde a amostra será selecionada.

Assim, podemos considerar que os erros de cobertura são devidos ao desencontro entre o objeto (unidade de análise, públicoalvo ou população que se deseja estudar) e o recorte: parte do objeto-alvo (tanto pessoas como coisas que se queira estudar) que é possível identificar e incluir na pesquisa. Um exemplo claro desse problema é a pesquisa residencial probabilística que é limitada por locais sem acesso ao entrevistador, como áreas violentas ou condomínios fechados, tais como prédios etc., onde as pessoas tinham sua participação limitada na maioria dos projetos de pesquisa, incluindo o senso nacional. Dessa maneira, novos meios de seleção e contato com potenciais participantes de uma pesquisa possibilitam diminuir esse erro de cobertura quando facilita a sua inclusão na amostragem ou aumentá-lo quando dificulta a participação.

Dessa forma os erros associados à cobertura acabam por trazer os erros de amostragem, i.e., pequenos vieses na seleção da amostra que podem ser leves e irrelevantes ou grandes e importantes e, dependendo do caso, permitir ou inviabilizar 
ANO 13 • NÚMERO 25 • 2으. SEM. 2016 • ORGANICOM

NOVOS MEIOS E NOVOS MÉTODOS DE PESQUISA: DESAFIOS, SOLUÇÕES E AVANÇOS

totalmente uma pesquisa. Por exemplo, pesquisas longitudinais de conteúdo publicitário estão hoje mais viáveis do que algum tempo atrás, devido aos bancos de dados criados por empresas especializadas (ex. arquivo da propaganda), somente possíveis devido à presença de computadores, softwares especializados e suas conexões; mais ainda, esses dados podem ser obtidos por qualquer indivíduo em qualquer parte do globo pelo acesso da internet; esta também facilita que as pesquisas internacionais tenham uma melhor cobertura, superada a barreira da língua.

Uma vantagem adicional, dentro dos aspectos de cobertura e que estão associados aos meios, é poder utilizar as características de cada um na obtenção da melhor amostra possível para o problema a ser pesquisado. Uma pesquisa quantitativa com visitantes de um ponto turístico no Canadá (GRAY, 2016) observou que mesmo utilizando um único método, questionário online, o direcionamento das entrevistas, também chamado criação de tráfico, é beneficiado por múltiplos pontos de contato, 0 que pode melhorar a distribuição da amostra, portanto a cobertura, mesmo para estudos qualitativos.

As pesquisas qualitativas que dependem de boas indicações de participantes, muitas vezes realizadas por empresas específicas de recrutamento, têm a necessidade de incluir pessoas que conheçam e/ou se interessem por determinado assunto. Nesse sentido, podem se beneficiar das mídias sociais digitais como fonte para a seleção, diminuindo o erro de cobertura, pois estas ampliam e especificam a população disponível para recrutamento, com vantagem da afinidade, ou seja, reunir pessoas a partir dos filtros determinados no projeto e no planejamento da pesquisa. A participação em grupos de discussão na web de determinados temas (uma espécie de netnografia) pode ser uma forma de detectar indivíduos que tenham o perfil idealizado para uma determinada pesquisa.

Dados disponíveis para acesso público, como em grupos de discussão, podem ser as fontes para, a partir das discussões publicadas, identificar indivíduos que possam participar desses projetos e que estejam dentro das necessidades definidas pelo pesquisador, ampliando assim a cobertura da população a ser observada; não se fala aqui de amostra probabilística, mas de uma inclusão maior e mais específica somente possível pelo acesso a internet. Nas comunidades on-line as pessoas antecipam suas necessidades e preocupações em relação a determinado tema. Esses dados combinados e associados a determinados indivíduos facilitam 0 recrutamento de pessoas que possam colaborar mais intensamente nas discussões em grupo ou em pesquisas em profundidade, além do quê analisar esses dados permite estabelecer pontos básicos como complemento aos temas para estas discussões.

De certa forma, ainda que tenhamos que lidar com a redução da população coberta pelos meios eletrônicos e digitais de comunicação, pode-se afirmar que, exceto no caso das pesquisas quantitativas que demandem uma amostragem probabilística pura, os diversos usos e aplicações desses meios, principalmente com múltiplos moldes, permitem uma amostragem mais bem distribuída da população, ou seja, com um alcance maior e uma cobertura melhor.

\section{Efeitos nas respostas}

Como vimos, a pesquisa de mercado trata da obtenção de informação necessária para a análise de determinado problema. Existe uma grande preocupação sobre a qualidade dessa informação e, nesse sentido, facilitar o trabalho do informante é objetivo maior do desenho da coleta de dados. Dados ruins geram, em geral, resultados ruins.

As pesquisas quantitativas foram as primeiras a se beneficiar dos novos meios disponíveis para coletar dados. Os chamados Cati (Computer assisted telephone interview) e Casi (Computer assisted self interview) foram os primeiros sistemas que, desenvolvidos nos anos 1980, permitiam a realização de surveys ao mesmo tempo em que o dado era armazenado e formatado 
ANO 13 • NÚMERO 25 • 2으. SEM. 2016 • ORGANICOM

NOVOS MEIOS E NOVOS MÉTODOS DE PESQUISA: DESAFIOS, SOLUÇÕES E AVANÇOS

em tabelas. Inicialmente a característica mais relevante desse método foi substituir o questionário em papel e as suas limitações. Os softwares desenvolvidos nesse período já ofereciam muitas vantagens na aplicação dos questionários, como utilizar cores diferentes para perguntas e respostas, a randomização de perguntas, a randomização de respostas, a utilização de dados da própria entrevista para construir perguntas (utilizar marcas citadas para construir uma pergunta sobre as marcas preferidas) e obviamente a formatação de banco de dados.

Os sistemas atuais (exemplos podem ser: SurveyMonkey, Qualtrix, GoogleDocs e outros) já vão muito além dessas características, incluindo a possibilidade de adicionar imagens e vídeos que sejam de interesse da pesquisa, medidas quase contínuas nas perguntas de escalas likert ou diferencial semântico através dos denominados sliders, ou seja, movimentar livremente uma barra dentro da escala sem a necessidade de marcar pontos fixos, medir o tempo que o entrevistado utilizou para responder cada questão etc.

Alguns outros aspectos podem ser considerados vantagens para o uso dos meios eletrônicos na obtenção de dados. Como, por exemplo, a complexidade do questionário transparente para o entrevistado - "pulos" (ex. quem responder "sim" pula para pergunta X) são dirigidos automaticamente, tanto no modelo com entrevistador (Cati) quanto no sem entrevistador (Casi); da mesma forma inconsistências (ex. ano de nascimento maior do que o aceito para a entrevista) são identificadas e resolvidas sem interferência e sem demora, da mesma forma como não-resposta ou esquecimento de responder podem gerar alertas para o respondente ou entrevistador e ser resolvidos imediatamente. Alguns estudos indicaram que existe uma confiabilidade maior no anonimato, principalmente no público jovem, e que as respostas "politicamente corretas" (aquelas que sofrem pressão social) acontecem em menor número nos meios eletrônicos, mas estes são dados que carecem de validação para os dias atuais e principalmente para a população brasileira.

Respostas também são influenciadas pela inclusão de imagens. Vera Toepel e Mick P. Couper (2011) demonstraram que a inclusão de imagens de comportamentos mais frequentes (como, por exemplo, ida ao supermercado é mais frequente do que ir ao shopping) fez os respondentes declararem frequências mais altas de outros comportamentos (ir às compras, por exemplo). Embora, no estudo desses autores, isso pudesse ser controlado pelas instruções verbais, é importante considerar que uma imagem inserida em uma pesquisa tem grande potencial de influenciar as respostas dadas pelos entrevistados. Ao mesmo tempo, ela pode ter um efeito de tornar a tarefa de responder à pesquisa mais agradável e com menos barreiras, uma vez que as imagens penetram pelos nossos sentidos antes mesmo da nossa mente tomar consciência, o que em semiótica chamamos de qualidade de sensação ou primeiridade (PEIRCE, 1977). Assim, as inserções de imagens, embora apresentem grandes vantagens na aplicação de questionários, oferece uma fonte de erro que deve ser considerada fortemente no desenho de questionários para pesquisas on-line. Embora imagens pudessem ser inseridas nos questionários off-line impressos por meio do uso de cartões, uma prática bastante comum, isso era custoso e de qualidade inferior, os meios eletrônicos permitem até inserir uma imagem em 3D e que gire na tela do respondente para, por exemplo, a avaliação de embalagens ou de design de produto.

A área de pesquisa qualitativa, onde inicialmente a grande revolução trazida pela internet foi a inclusão de chats na coleta de dados, evoluiu consideravelmente com as novas tecnologias e, principalmente, novos softwares especializados nos diversos métodos utilizados, sendo hoje talvez a área que oferece a maior diversidade nas formas de obter informação. Um ponto importante a ser considerado para esse desenvolvimento foi o incremento nas habilidades de digitação da população causada pela sua larga utilização nas novas formas de comunicação (Facebook, Whatsapp, Instagram, Snapchat e-mail etc.). 0 conforto com a digitação oferece uma oportunidade para que os participantes teçam suas observações digitando-as, o que permite que o pesquisador possa obter diversos tipos de dados automaticamente, sem necessidade de gravação e transcrição; além disso, a fala (verbal ou escrita) de um participante pode ser usada como estímulo em outros grupos de forma totalmente natural. Outra 
ANO 13 • NÚMERO 25 • 2으. SEM. 2016 • ORGANICOM

NOVOS MEIOS E NOVOS MÉTODOS DE PESQUISA: DESAFIOS, SOLUÇÕES E AVANÇOS

vantagem é que os participantes também desenvolveram habilidades relacionadas às imagens, tanto de produção, quanto de buscas em bancos de dados imagéticos, o que permite exercícios na rede como simulacros das colagens e demais técnicas projetivas largamente utilizadas nas discussões em grupo presenciais.

Da mesma forma que nos métodos quantitativos, temos hoje uma possibilidade maior de oferecer estímulos visuais nas pesquisas qualitativas como forma de provocação e, valendo-nos do fato de que os participantes não precisam estar no mesmo local, podemos ter, dentro de um mesmo grupo, indivíduos que receberam estímulos diferentes se isso for de interesse da pesquisa. 0 fato de não estar no mesmo local oferece ainda a possibilidade de anonimato mesmo em grupos que estão interagindo ao mesmo tempo. Essa característica é particularmente importante quando os temas de investigação envolvem tabus ou mesmo preconceitos e áreas como higiene e beleza, todas sensíveis no aspecto da reprovação/aprovação social.

Com essas modificações, novos termos foram adicionados aos métodos, tais como síncrono (ao mesmo tempo) e assíncrono (sem necessidade de ser ao mesmo tempo). Dessa forma, discussões em grupo realizadas via email, bulletin boards etc., que permitem participação em diferentes horários e locais, são do segundo tipo e as realizadas via Skype, Facetime e assemelhados, por exemplo, mais perto das formas tradicionais, são consideradas como discussões em grupo virtual e pertencem ao primeiro grupo. Mais recentemente, observamos a ampliação do uso do Whatsapp como instrumento de pesquisa, prioritariamente qualitativo. Facilitado pela expansão dos aparelhos celulares, do tipo smartphone, este aplicativo de conversa em tempo real traz imensa agilidade às pesquisas. Evidentemente, não é rentável para qualquer tipo de pesquisa, mas pode ser um instrumento adicional importante ou mesmo aquele acessível quando não há outras possibilidades. A questão central do uso de Whatsapp está na seleção e formação do grupo. A afinidade e a disposição de participação é fundamental (mas, também 0 é em qualquer técnica). Como o aplicativo permite troca de mensagens de texto, mas também inclusão de imagens e filmes, amplia as possibilidades de uso de variados estímulos, além da reação imediata a eles nas pesquisas de mercado.

Algumas vantagens da inclusão dos novos meios na pesquisa qualitativa foram identificadas por Franklin e Lowry (2016), por exemplo, nas situações onde o participante insere suas respostas diretamente no computador (por voz ou digitação); notouse que a qualidade das respostas aumenta pela facilitação da entrada dos dados e ausência da necessidade de transcrição, já que as vozes ou os textos ficam automaticamente disponíveis para análise. Essa facilidade, que é notadamente útil para os métodos mais tradicionais, como entrevista em profundidade e discussão em grupo, colabora muito para técnicas associadas à análise de dados qualitativos, como por exemplo, grounded theorye Delphi studies, que necessitam de uma grande e rápida interação de dados qualitativos, o armazenamento automático desses dados e sua interação com softwares tais como Atlas.ti e Nvivo, permitindo que as pesquisas sejam mais rápidas e com maior opções para a sua análise.

Assim, como observado nas pesquisas quantitativas, os novos meios trazem consigo alguns riscos, bem apontados por Kathy $\mathrm{K}$. Franklin e Catherine Lowry (2016), tais como a dificuldade observada por moderadores para o direcionamento ou a exploração de um ponto de interesse em discussões em grupo com a utilização de digitalização pelos participantes, pois a velocidade de interação entre eles aumenta bastante a quantidade de informação a ser processada pelo moderador, aspecto este que é facilmente controlado na situação off-line. A própria habilidade do moderador com essa forma de interação (a digitalização) pode ser uma grande barreira para sua utilização, assim como as diferentes velocidades dos participantes podem privilegiar alguns e prejudicar a participação de outros; embora similar ao que possa acontecer nas discussões em grupos tradicionais em relação à habilidade verbal, isso também é facilmente controlável pelo moderador nos meios off-line.

Embora semelhantes aos dados transcritos da discussão presencial, os dados digitados diretamente pelo participante são, em geral, muito mais concisos e específicos, tornando a tarefa de moderação e a posterior análise muito mais intensa, pois 
ANO $13 \cdot$ NÚMERO 25 • 2은. $2016 \cdot$ ORGANICOM

NOVOS MEIOS E NOVOS MÉTODOS DE PESQUISA: DESAFIOS, SOLUÇÕES E AVANÇOS

cada frase contém, potencialmente, uma quantidade maior de informação de interesse do projeto. Por outro lado, muitas frases podem estar incompletas ou truncadas e vão necessitar uma grande capacidade interpretativa do analista para seu aproveitamento na codificação e interpretação das informações.

\section{EFEITOS ASSOCIADOS AO MÉTODO}

A interação entre a tecnologia e a metodologia de pesquisa pode ter como resultado alguns aspectos que, embora sejam causados pelo formato no qual a tecnologia se apresenta, pertencem de fato aos métodos, ou seja, não são associados especificamente aos meios, mas ao método escolhido pelo pesquisador. Por exemplo, o uso de pesquisas quantitativas pela internet traz consigo as vantagens e desvantagens das técnicas das autoentrevistas que estarão presentes em qualquer meio que faça uso dessa técnica. Assim, na sua grande maioria, questionários que são respondidos (na internet ou fora dela) sem a presença de um entrevistador podem abordar temas mais íntimos; podem ainda ser respondidos no momento e local que melhor convierem ao respondente; mas podem sofrer com perguntas mal respondidas, incompletas e/ou mal compreendidas. Também não se pode garantir que o respondente seja a mesma pessoa que foi selecionada para a amostra (pesquisas com executivos podem muitas vezes ser respondida por um assistente) e nem que as respostas representem as suas opiniões isoladamente da influência de outros. Assim, todos os meios que utilizam essa técnica estão sujeitos a esses aspectos.

No entanto, o meio digital traz consigo algumas soluções para esses velhos problemas. Com as diversas possibilidades de interação oferecidas pela internet, dúvidas, informações e orientações podem ser disponibilizadas on-line, ou mesmo através de robôs que farão o papel de entrevistador ou tira-dúvidas, como já se observa hoje em sites de empresas de telefonia celular, a exemplo da Vivo, ou de varejo, como é o caso da Magazine Luiza. Em casos onde exista a necessidade de identificação ou isolamento do respondente, as web-câmeras podem ser de grande ajuda, evitando a substituição do respondente.

No caso das pesquisas qualitativas, essa associação entre tecnologia e metodologia é menos limitante do que no caso das quantitativas. Um problema resultante dessa interação está associado com a objetividade que se busca nas discussões em grupo, uma grande preocupação nesse método, que pode ser exacerbada na forma on-line pela dificuldade de direcionamento e interferência do moderador. Isso pode em alguns casos prejudicar a recolha e compreensão das informações de grupos específicos, pela perda do direcionamento aos objetivos da pesquisa, e em outros reduzir o viés implícito do moderador nesse direcionamento.

Assim, a seleção de um método de pesquisa deve antes considerar os aspectos técnicos associados a ele, para então ter em conta como os meios de coleta de dados e informações podem minimizar seus pontos negativos e se beneficiar de suas vantagens, fazendo evoluir os resultados e as potencialidades dos projetos de investigação.

\section{ASPECTOSÉTICOS}

A integração de novos meios para recolha e análise de dados e informações traz consigo a preocupação com cuidados antigos, que podem ser exacerbados, e novas questões que são inerentes aos meios inaugurais e por isso merecem ser observados dentro de suas especificidades. 
ANO 13 • NÚMERO 25 • 2으. SEM. 2016 • ORGANICOM

NOVOS MEIOS E NOVOS MÉTODOS DE PESQUISA: DESAFIOS, SOLUÇÕES E AVANÇOS

Algumas práticas que já são tradicionalmente condenadas eticamente, como o uso de identificação do respondente e sua utilização para as atividades de marketing da empresa contratante, podem ser muito facilitadas pelos meios eletrônicos e digitais que possuem uma capacidade maior de armazenamento, processamento e memória. Enquanto que nas formas tradicionais se dependia do desejo expresso do respondente para a obtenção das informações, nos meios on-line isso é tremendamente facilitado e pode ser obtido sem o consentimento, o que já é uma prática usual em ações de marketing na internet. Por outro lado, em alguns projetos os participantes são autosselecionados por clicarem em um link disponibilizado em páginas específicas na internet, normalmente sites dos clientes, causando uma grande dificuldade na identificação do indivíduo e na verificação das respostas dadas, facilitando as fraudes. Sabe-se que essa prática de verificação nas pesquisas tradicionais presenciais atinge por vezes $30 \%$ da amostra, garantindo confiabilidade e boa qualidade de dados e informações.

A participação de "profissionais" de pesquisa (HILLYGUS; JACKSON; YOUNG, 2014) - comportamento já observado nas formas tradicionais, i.e., pessoas que respondem à pesquisa por interesse financeiro - está muito mais desenvolvida nos meios eletrônicos, devido ao grande número de empresas que hoje recrutam esses indivíduos, oferecendo compensação financeira pela participação. Esse é um problema que está no limite da ética, pois, ao mesmo tempo em que se observa uma grande vantagem na coleta de dados mais rápidos e mais específicos, pesquisas realizadas em outros países indicaram que existe uma tendência de que a frequência de participação afeta a qualidade dos dados (COEN; LORCH; PIEKARSKI, 2005); seja pela fraude de informação, seja pela experiência adquirida pelo respondente com a intensa participação, ele vira uma espécie de "profissional respondente". Ainda que existam meios para controlar isso, como associações de classe que mantêm banco de dados de nomes e registros de identidade dos participantes, é notório que a "profissionalização da resposta" na internet fica muito facilitada pelos múltiplos meios de acesso que os indivíduos podem usar.

Os meios eletrônicos facilitam fraudes que antes nunca tinham sido observadas como, por exemplo, simular uma discussão em grupo copiando uma discussão de um bulletin board, ou em pesquisa participante onde o entrevistador não se identifica nem antes, nem durante e nem depois das entrevistas, violando o direito dos demais participantes em ter seus dados utilizados sem sua autorização expressa. Outro ponto que é novo nos cuidados com a pesquisa on-line é a disponibilidade eletrônica dos dados, que podem ser acessados ilegalmente por indivíduos mal-intencionados e ser usados para funções diferentes daquela para a qual foram coletados.

\section{CONSIDERAÇÕES FINAIS}

Após o percurso reflexivo apresentado, observa-se, portanto, que o desenvolvimento de novas formas de comunicação e de suas linguagens próprias afeta diretamente a prática da pesquisa de mercado, trazendo vantagens e desvantagens que devem ser foco de atenção de profissionais e pesquisadores acadêmicos. Compreender a potencialidade das tecnologias digitais possibilitará a apropriação de novas técnicas e métodos de investigação, sem, no entanto, deixar de ter em conta suas limitações e seus desafios, uma vez que todo método impõe limites.

Nesse sentido, nossa atenção deve ser dirigida para os efeitos apontados neste trabalho e outros que, por limitação das reflexões aqui demonstradas ou por ainda não terem sido observados pelos estudiosos e profissionais da área, possam afetar a qualidade e a reputação da pesquisa de mercado. Mais ainda, cabe aos pesquisadores da área a missão de descobrir novas e vantajosas aplicações dos rápidos desenvolvimentos tecnológicos observados no campo da comunicação, ampliando com isso a capacidade de entendimento dos aspectos relacionados aos produtos, às empresas, às marcas e também aos serviços e aos indivíduos, considerando suas formas, conteúdos, intenções e comportamentos. 


\section{REFERÊNCIAS BIBLIOGRÁFICAS}

CANEVACCI, Massimo. Fetichismos visuais. São Paulo: Ateliê, 2008.

COEN, Terence; LORCH, Jacqueline; PIEKARSKI, Linda. The effects of survey frequency on panelists' responses. In: Worldwide panel research conference ESOMAR, Amsterdam, 2005.

FRANKLIN, Kathy K.; Lowry, Catherine. Computer-mediated focus group sessions: naturalistic inquiry in a networked environment. Qualitative Research, v. 1, n. 2, p. 169-184, 2001.

GRAY, Shannon. The changing world of destination market research: a comparison of methods utilized in driving traffic to an online survey. August 18, 2016. Tourism Travel and Research Association: Advancing Tourism Research Globally. Paper 69. Disponível em: <http://scholarworks.umass.edu/ttra/2011/0ral/69>.

HALL, Stuart. A identidade cultural na pós-modernidade. São Paulo: DP\&A, 2004.

HILLYGUS, D. Sunshine; JACKSON, Natalie; Young, McKenzie. Professional respondents in non-probability online panels. In: CALLEGARO, Mario et. Al. (Orgs.). Online panel research: a data quality perspective, John Wiley \& Sons, 2014. 219-237.

JENKINS, Henry. Cultura da convergência. São Paulo: Aleph, 2009.

JENKINS, Henry; FORD, Sam; GREEN, Joshua. Cultura da conexão. São Paulo: Aleph, 2015.

LIPOVETSKY, Gilles. O império do efêmero. São Paulo: Cia. das Letras, 2008.

PEIRCE, Charles S. Semiótica. São Paulo: Perspectiva, 1977.

PEREZ, Clotilde. Ser ou não ser pesquisador, eis a questão! PMKT: Revista Brasileira de Pesquisas de Marketing, Opinião e Mídia, v. 13, p. 35, 2015.

Signos da marca. São Paulo: Cengage, 2004.

TOEPOEL, Vera; COUPER, Mick P. Can verbal instructions counteract visual context effects in web surveys? Public Opinion Quarterly, v. 75, n. 1, p. 1-18, 2011.

Artigo recebido em 01.10.2016 e aprovado em 09.12.2016. 